\title{
Defecatory urge increases cognitive control and intertemporal patience in healthy volunteers
}

\author{
Dongxing Zhao ${ }^{1,2}$ (D) | Maura Corsetti ${ }^{3,4}$ (i) | Mehrad Moeini-Jazani ${ }^{5}$ | \\ Nathalie Weltens $^{1}$ | Mirjam Tuk ${ }^{6,7}$ | Tack Jan ${ }^{1}$ | Luk Warlop ${ }^{8}$ | Lukas Van Oudenhove ${ }^{1}$ \\ ${ }^{1}$ Translational Research Centre for Gastrointestinal Disorders (TARGID), KU Leuven, Leuven, Belgium \\ ${ }^{2}$ Institute for Diabetes Research and Metabolic Diseases (IDM) of the Helmholtz Center Munich at the University of Tübingen, Tübingen, Germany \\ ${ }^{3}$ NIHR Nottingham Biomedical Research Centre (BRC), Nottingham University Hospitals NHS Trust and the University of Nottingham, Nottingham, UK \\ ${ }^{4}$ Nottingham Digestive Diseases Centre, School of Medicine, University of Nottingham, Nottingham, UK \\ ${ }^{5}$ Faculty of Economics and Business, University of Groningen, Groningen, The Netherlands \\ ${ }^{6}$ Imperial College Business School, London, UK \\ ${ }^{7}$ Rotterdam School of Management, Erasmus University, Rotterdam, The Netherlands \\ ${ }^{8}$ Department of Marketing, BI Norwegian Business School, Oslo, Norway
}

\section{Correspondence}

Lukas Van Oudenhove, Laboratory for BrainGut Axis Studies (LaBGAS), Translational Research Centre for Gastrointestinal Disorders (TARGID), KU Leuven, B-3000 Leuven, Belgium.

Email: lukas.vanoudenhove@kuleuven.be and

Luk Warlop, Department of Marketing, BI Norwegian Business School, N-0484 Oslo, Norway

Email: luk.warlop@bi.no

Funding information

The work was supported in part by a grant from FWO Flanders (Grant No. G.0.396.10.N.10) to Luk Warlop and by a grant (STRT/12/002) from the KU Leuven Special Research Fund to Lukas Van Oudenhove.

\begin{abstract}
Background: Past research has demonstrated that moderate urge to urinate improves inhibitory control, specifically among participants with higher behavioral inhibition sensitivity (BIS). The effect was absent when the urge exceeded intolerable level. The present research examines whether rectal distension-induced urge to defecate has similar effects.

Methods: The moderate and high defecatory urge were induced by rectal distension in healthy volunteers $(n=35)$, while they completed Stroop task and monetary delay discounting task. The difference of average reaction time between incongruent and congruent trials in the Stroop task (Stroop interference) and the preference for largerlater rewards in the delay discounting task were the primary outcomes.

Key Results: Participants with high BIS $(n=17)$ showed greater ability to inhibit their automatic response tendencies, as indexed by their Stroop interference, under moderate urge relative to no urge $\left(128 \pm 41 \mathrm{~ms}\right.$ vs $202 \pm 37 \mathrm{~ms}, t_{64}=2.07 ; P=0.021$, Cohen's $d$ : 0.44$)$, but not relative to high urge $\left(154 \pm 45 \mathrm{~ms}, t_{64}=1.20 ; P=0.12\right.$, Cohen's $d: 0.30$ ). High BIS participants also showed a higher preference for largerlater reward in the delay discounting task under high (odds ratio $=1.51$ [1.02-2.25], $P=0.039$ ) relative to no urge, but not relative to moderate urge (odds ratio $=1.02$ [0.73-1.42], $P=0.91$ ). In contrast, rectal distension did not influence performance on either of the tasks in participants with low BIS $(n=18)$.
\end{abstract}

Abbreviations: AIC, Akaike's information criterion; aMCC, anterior midcingulate cortex; BAS, behavioral activation system; BIS, behavioral inhibition system; IBS, irritable bowel syndrome; MDP, minimal distending pressure; $\mathrm{mPFC}$, medial prefrontal cortex; pACC, pregenual anterior cingulate cortex; RT, reaction time; VAS, visual analogue scale. 
Conclusions and inference: These findings may be interpreted as a "spill-over" effect of inhibition of the urge to defecate to volitional cognitive control among healthy participants with high BIS.

\section{KEYWORDS}

defecatory urge, gut-brain axis, inhibitory control, rectal barostat

\section{1 | INTRODUCTION}

Inhibitory control refers to one's ability to restrain and override impulses to achieve goals of higher importance or with better payoffs. ${ }^{1,2}$ A growing body of literature highlights the existence of a common, domain-independent capacity for inhibitory control. ${ }^{3,4}$ Specifically, when inhibitory control is activated in one domain, it can facilitate control in other (unrelated) domains. For instance, controlling the urge to urinate facilitates inhibitory control in cognitive domains, specifically in tasks requiring impulse inhibition. ${ }^{2}$ It has been proposed that inhibitory control processes across different domains share common neurological pathways, ${ }^{5}$ involving brain regions along the cingulate sulcus, with dopamine being the key neurotransmitter. ${ }^{6,7}$

Neurological processes underlying rectal filling are in many ways similar to bladder control processes. ${ }^{5,8-10}$ Consequently, in the present research, we propose that defecatory urge should also facilitate inhibitory control. We explore this hypothesis across two self-regulatory tasks by examining the interplay between rectal urge conditions of varying degrees and individuals' chronic differences in behavioral inhibition sensitivity (BIS). ${ }^{11}$ Specifically, we hypothesized that, for people with high BIS, experiencing defecatory urge, relative to no urge, should improve performance on tasks that require inhibitory control. Past research shows that increased urination urge reportedly enhanced inhibitory control only among individuals with high BIS sensitivity. ${ }^{2}$ This is consistent with reinforcement sensitivity theory ${ }^{12,13}$ which argues that BIS fosters inhibition of prepotent conflicting behaviors and facilitate self-regulation. Therefore, we expected the positive consequences of controlling defecatory urge on self-regulation to be limited to participants with high BIS.

Following past research, we chose the Stroop task and delay discounting task to measure inhibitory control. ${ }^{14}$ In particular, the Stroop task ${ }^{15}$ is a robust and well-established method to measure cognitive control. It measures people's ability to disregard distractions and override impulses in the service of task performance. ${ }^{16,17}$ Moreover, delay discounting measures one's ability to resist immediate temptations and to wait for larger-later rewards, signifying patience, and self-control. Both of these tasks are commonly used as measures of inhibitory control.

Finally, we used rectal distension technique (barostat) to induce varying levels of defecatory urge. This is a widely used technique, which enables induction of rectal sensation in a systematic and in tensity-controlled manner. This is important because while past

\section{Key Points}

- Defecatory urge improves inhibitory control in healthy volunteers.

- Inhibition of the urge to defecate facilitated cognitive control under moderate urge, and increased preference for larger-later rewards in the delay discounting task under high urge, among healthy humans with high behavioral inhibition sensitivity (BIS), but not among those with low BIS.

- These results demonstrate that mechanical signals originating in the rectum that require inhibition can improve inhibitory control in other domains, and contribute to studies on cognitive function in functional GI disorders.

research on bladder filling has found that inhibition of urination urge facilitates cognitive control, ${ }^{2}$ other findings suggest that extreme urination urge may impair cognitive functioning. ${ }^{18}$ Using a controlled rectal distension method, therefore, enables us to test our hypothesis across different levels of defecatory urge (eg, no, medium, and high urge) and to explicate potential boundaries of our hypothesis.

\section{2 | MATERIALS AND METHODS}

\section{1 | Participants}

Healthy male, and non-pregnant, non-breastfeeding female participants, aged 18-60 years old at the time of consent, were recruited in this randomized, counterbalanced, single-blinded mechanistic interventional crossover study. Candidates with color blindness or those who used any medication or drugs (except contraceptive pills) were excluded. All study visits were conducted in the endoscopy unit, at University Hospital Gasthuisberg of the KU Leuven. The sample size was calculated based on the previous findings ${ }^{2}$ showing a medium-sized effect of moderate urinary urge on Stroop performance in high BIS participants (one-tailed paired $t$ test, alpha $=0.05$, Cohen's $d=0.50$, power $=85 \%$ ). Last menstrual period from female volunteers was recorded during screening, and all study visits were scheduled during the follicular phase. A schematic overview of the study is presented in Figure 1. 
FIGURE 1 Schematic overview of the experimental procedure. (A) overview of a study visit: firstly, a threshold determination involving a stepwise distension session was undertaken, followed by tonic distension blocks to ensure that the stimulus length would allow stable pressure, volumes, and ratings of moderate/high urge. Finally, three test blocks were performed (one for each level of urge) in a counterbalanced order. (B) overview of a test block: firstly, instructions were provided, and a Stroop practice session was undertaken, followed by 30 Stroop trials in a randomized order, followed by five delay discounting tasks. At the end of each test block, retrospective ratings of urge and pain were collected using visual analogue scales (VAS). (C) an example of a Stroop trial: presented in the following sequence: a fixation cross displayed $500 \mathrm{~ms}$, followed by a blank space for $500 \mathrm{~ms}$, followed by stimulus presentation for $2000 \mathrm{~ms}$, followed by an intertrial blank space whose display time varied between 1500 and $3000 \mathrm{~ms}$ (uniform distribution) until the next trial

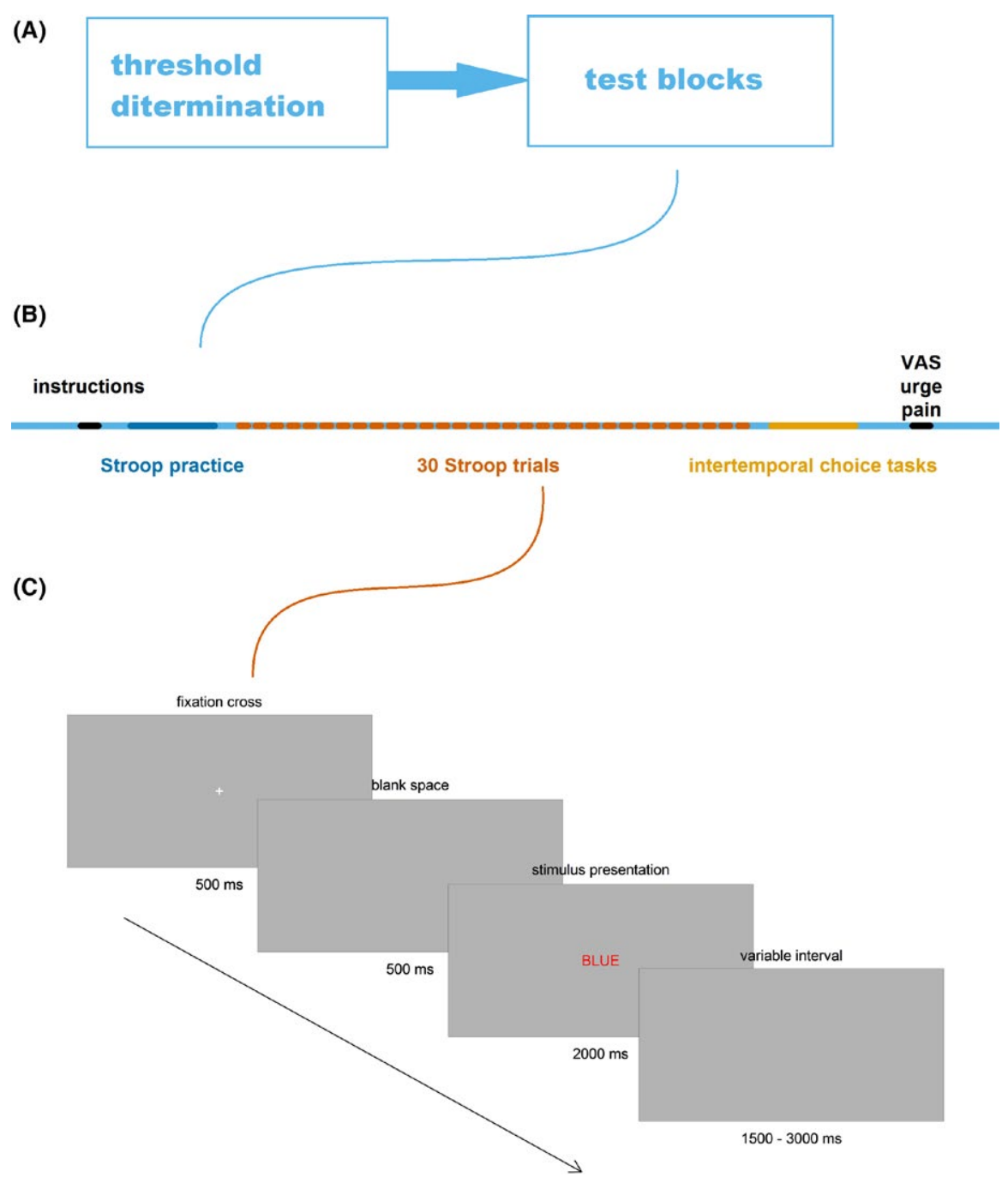

\subsection{Ethical approval and clinical trial registration}

The study was approved by the Medical Ethics Committee of the University Hospitals Leuven; Belgium (ML10139, 17 Aug 2014), preregistered at ClinicalTrials.gov (NCT02043561: https://clinicaltrials.gov/, 23 Jan 2014), and performed in accordance with the Declaration of Helsinki, including written informed consent.

\section{3 | Behavioral Inhibition Sensitivity}

The Behavioral Inhibition Sensitivity/Behavioral Activation Sensitivity scale (BIS/BAS) ${ }^{11}$ was administered together with screening questionnaires before participants' visit to the laboratory. Participants were divided into two groups (high BIS group vs low BIS group) based on a median split on the BIS scale to test our hypothesis that the effect of urge inhibition would occur in high BIS participants only.

\section{4 | Distending device}

Rectal distensions were induced by an intrarectal balloon $(600 \mathrm{~mL}$ capacity) and controlled by an electronic barostat (Dual Drive
Barostat, Distender Series II; G\&J Electronics Inc). A personal computer running custom-made software provided by the manufacturer (Protocol Plus, G\&J Electronics Inc) was used to program the barostat and to record the intraballoon pressure and volume continuously.

\section{5 | Preparation}

Following an overnight fast and rectal cleansing with tap water enema, a finely folded balloon was introduced into the participants' rectum with the caudal end $6 \mathrm{~cm}$ from the anal verge and then connected to the barostat device. Participants were placed in a semi-recumbent position on a bed. The balloon was then unfolded by inflation with $150 \mathrm{~mL}$ of air and after that completely deflated.

\section{6 | Threshold determination}

The minimal distending pressure (MDP) was first determined, as the pressure level at which respiratory fluctuations were regularly recorded. To determine the individual threshold for defecatory urge, 
a stepwise pressure-controlled distension procedure (steps of $4 \mathrm{~mm}$ $\mathrm{Hg} / 30 \mathrm{~s}$ ) starting from MDP was performed, ${ }^{19,20}$ following a 15-minute accommodation period. After 15 seconds into each distension step, participants rated their perceived intensity of defecatory urge and pain, using a 100-mm visual analogue scale (VAS) ranging from "no urge" to "maximal urge" and "no pain" to "maximal pain" on a computer screen. The stepwise distension procedure was ended when either the participants reported maximal urge (100 on the VAS) or an intraballoon volume of $500 \mathrm{~mL}$ was achieved.

Three pressure thresholds were derived from this stepwise distension sequence ${ }^{20}$ and were used during the subsequent parts of the study:

1. No urge (balloon deflated)

2. Moderate urge (first pressure step evoking a score of 41 or more on the urge VAS)

3. High urge (first pressure step evoking a score of 81 or more on the urge VAS)

After the stepwise distension, participants went through three tonic distension blocks, in which they were exposed to tonic distension at no urge, moderate urge, and high urge pressure thresholds (in counterbalanced order) for 180 seconds and were asked to rate their urge and pain sensations on a VAS every 30 seconds (to ensure that this stimulus length would allow us to achieve stable pressures, volumes, and above all ratings of moderate/high urge).

\section{7 | Test blocks}

Between the threshold determination and the test blocks, participants rested in bed for 10 minutes while the rectal balloon was not extubated. During each test block, rectal distension at one of the three individually titrated pressure thresholds, described above, was administered in counterbalanced order, with a 10-minute break in between. During each block, participants performed the Stroop task followed by the delay discounting task. At the end of each block, all participants rated VAS for urge and pain retrospectively.

\subsection{1 | Stroop task}

The Stroop task requires participants to indicate the font color of a series of visually presented color words as quickly as possible. In incongruent trials, participants should ignore the meaning of the color word, thus override their dominant and impulsive response tendencies, and attend to its font color instead. Stroop performance is assessed by calculating the difference between the average response latencies in incongruent and congruent trials, an index referred to as Stroop interference. A smaller Stroop interference score, therefore, indicates greater cognitive ability to disregard distractions and impulses and to stay focused on the goal.

The Stroop task consisted of three types of trials ${ }^{21}$ : the "word naming" trials in which the color words (eg, the word RED) were displayed in black font, and the "incongruent" trials (eg, the word RED in blue font) and the "congruent" trials (eg, the word RED in red font) in which the color words were displayed in colors. For congruent and incongruent stimuli, participants were instructed to neglect the meaning of the word, but only respond to the font color of the displayed stimuli. To respond across these trials, participants pressed one of three keys on a keyboard labeled with colors red, blue, or green that corresponded to the word color (ie, in the word-naming trials) or the font color (ie, in the congruent and incongruent trials) of the stimuli.

A Stroop trial consisted of the following sequence: a fixation cross for $500 \mathrm{~ms}$, followed by a blank space for $500 \mathrm{~ms}$, and stimulus presentation for $2000 \mathrm{~ms}$ The intertrial intervals were uniformly distributed between 1500 and 3000 ms Responses were recorded between the start of stimulus presentation until $1500 \mathrm{~ms}$ into the intertrial interval; the stimulus disappeared from the screen as soon as the response was registered. Any response faster than $150 \mathrm{~ms}$ was considered abnormal and hence removed before the analysis. For each participant, the average reaction time for each trial type (ie, congruent, incongruent, and word naming) was then calculated. As mentioned earlier, the main dependent variable was Stroop interference which was calculated by subtracting average response times in congruent trials from the average response time in incongruent trials for each participant.

At the beginning of the experiment, participants went through one practice block in which the three trial types were presented with instructions. During the practice block, stimuli from the three trial types were presented in a fixed order: word naming, congruent, and incongruent. Afterward, at each distension level, participants performed a block of 30 Stroop trials, consisting of 10 trials of each type. The 30 trials at each distension pressure level were presented in a random order, and no feedback was provided. However, the practice block would repeat until participants responded to all 30 stimuli correctly. Colors were counterbalanced between trial blocks. Presentations were therefore unblocked and uncued within each trial block to ensure the maximal experience of conflict.

\subsection{2 | Delay Discounting Task}

Immediately after each Stroop trial, participants performed a delay discounting task, consisting of five intertemporal monetary choices. ${ }^{22}$ For each choice, participants indicated their preference for either a smaller, sooner or a larger, later monetary reward ${ }^{22}$ (eg, "Would you prefer 24 euro now or 35 euro in 29 days?"). Participants pressed button "1" on the keyboard to choose the immediate but smaller amount, and " 2 " to choose the larger but delayed amount. Participants' choices were then treated as binary outcomes in a logistic regression model. Overall, participants' tendency to choose larger, later rewards over smaller, sooner rewards signifies their ability to resist immediate temptations and to wait for larger rewards in the future, a critical capacity for self-regulation.

In the informed consent form, and upon completion of the three test blocks, all participants signed a document indicating that they 
would be awarded a fixed amount of money after completing the experiment.

\subsection{Statistical analysis}

All statistical analysis was done using SAS Version 9.4 (SAS Institute, Cary, NC, USA). Reaction times of the Stroop test were reported as mean \pm SEM. Differences were considered significant when $P \leq 0.05$. The variance-covariance structure providing the best fit was chosen based on the minimum value of Akaike's Information Criterion (AIC).

For each participant, response latencies within each Stroop condition (ie, congruent, incongruent, and word naming) were prepared in each test block using trimming methods ${ }^{23}$ and subsequently averaged. Specifically, for each participant, all the erroneous responses, responses faster than $150 \mathrm{~ms}$, and any responses 3 $\mathrm{SD}$ above or below the mean of the participant's response latencies per Stroop and urge condition were removed prior to calculating average response latencies. The remaining data were then averaged for each urge and Stroop condition, for each participant. The preprocessed Stroop color-naming data were then analyzed in a 3 (urge condition) $\times 3$ (Stroop condition) within-subject mixed model including the urge condition-by-Stroop condition interaction effect in each BIS group separately, with the order of the test blocks, and the subjective pain rating added as covariates. Lower-tailed planned $\mathrm{t}$-contrasts were performed to test our hypotheses that Stroop interference-the difference between average response latencies between incongruent and congruent trials-decreases under moderate (but not under high urge), compared to no urge condition, in the high BIS group, but not in the low BIS group. To examine the potential effect of urge condition on response latencies in general (ie, not related to inhibitory processes), response latencies in the Stroop word-naming condition were also analyzed in a separate mixed model. Specifically, two-tailed planned t-contrasts were performed to test our hypothesis that response latencies in the Stroop word-naming trials did not vary as a function of the urge for each BIS condition.

Participants' choice between a larger, later reward and a smaller, immediate reward in the delay discounting task was treated as a binary dependent variable in a generalized linear mixed model with urge condition as a within-subject factor, and order of the test block and subjective pain rating as covariates, ${ }^{24}$ in each BIS group separately. The probability of choosing a larger-later reward was further compared in high vs no urge condition, and moderate vs no urge condition for each BIS group.

\section{3 | RESULTS}

\section{1 | Participants}

Thirty-five healthy volunteers [23 (66\%) women, age: $22 \pm 0.3$ years, recruited between September 10, 2014 and September 3, 2015] participated in the study (Supplementary Figure S1). Participants were divided into high $(n=17)$ and low $(n=18)$ BIS groups, based on a median split of their BIS score (median [IQR] 22 [18, 23]). Four female volunteers in the low BIS group and one in the high BIS group self-reportedly took contraceptive pills. This proportion did not differ between high and low BIS groups (Fisher exact test $P=0.16$ ). The demographic characteristics of the participants are presented in Table 1. All participants completed the study, and there was no adverse event reported throughout the experiment. The overall error rate in the Stroop task was $2.32 \%$, and all participants completed the Stroop task with more than $90 \%$ correct answers. Therefore, no participant was excluded for making too many errors. Table 2 shows the overall error rates in the Stroop task in each urge condition. Further, statistical analysis was also performed on Stroop interference including the number of errors as a covariate, but the main outcomes did not change.

\section{2 | Manipulation check}

Personalized distension pressures (moderate urge: $29.6 \pm 1.2 \mathrm{~mm}$ $\mathrm{Hg}$, high urge: $41.6 \pm 1.1 \mathrm{~mm} \mathrm{Hg}$ ) were determined according to each participant's average subjective urge ratings on a $100-\mathrm{mm}$ scale. The average retrospective urge ratings, measured at the end of each test block, were significantly higher in the moderate $(41.0 \pm 3.3 \mathrm{~mm})$ and high $(67.2 \pm 4.2 \mathrm{~mm})$ urge conditions compared to the no urge condition $(20.0 \pm 3.3 \mathrm{~mm}$ ), as shown in Figure $2 \mathrm{~A}$ (main effect of urge condition, $F_{2,34}=52.65, P<0.0001$, planned contrast: moderate urge vs no urge, $t_{34}=5.08, p_{\text {Holm }}<0.0001$, high urge vs no urge, $t_{34}=9.48, p_{\text {Holm }}<0.0001$, high urge vs moderate urge, $t_{34}=7.82$, $\left.p_{\text {Holm }}<0.0001\right)$. These results confirm that our urge manipulation was successful and worked as intended.

Similarly, retrospective pain ratings, measured at the end of each test block, were significantly higher in the high urge $(27.3 \pm 4.9 \mathrm{~mm})$ compared to the no urge $(5.4 \pm 1.7 \mathrm{~mm})$ condition. However, no difference between the moderate urge $(11 \pm 2.9 \mathrm{~mm})$ and no urge conditions was found (main effect of urge condition, $F_{2,34}=14.76$, $P<0.0001$, planned contrast, moderate vs no urge, $t_{34}=1.90$ $p_{\text {Holm }}=0.15$, high vs no urge, $t_{34}=5.39 p_{\text {Holm }}<0.0001$, high vs moderate urge, $t_{34}=4.50 p_{\text {Holm }}=0.0002$ ), as shown in Figure $2 B$. Given
TABLE 1 Demographic characteristics of participants

\begin{tabular}{|llllc|}
\hline & High BIS group & Low BIS group & & P value \\
\hline Gender (F/M) & $12 / 5$ & $11 / 7$ & $\chi^{2}=0.097$ & 0.76 \\
\hline Age $(\mathrm{y})$ & $23.5 \pm 1.4$ & $22.2 \pm 0.4$ & $t=0.84$ & 0.41 \\
\hline BIS score & $23.7 \pm 0.4$ & $18.4 \pm 0.4$ & $t=9.18$ & $<0.0001$ \\
& [range: 22,28$]$ & [range: 16,21$]$ & & \\
\hline
\end{tabular}


the differences in pain ratings between the high urge and the other conditions, we controlled for pain ratings in all subsequent analyses.

\section{3 | Stroop interference}

\subsection{1 | High BIS group}

The personalized distension pressures were not associated with participants' Stroop interference in high BIS group $(P>0.05)$. Consistent with our hypothesis, the Stroop interference was significantly smaller in the moderate urge condition compared to the no urge condition (planned contrast, lower tailed, moderate urge $128 \pm 41 \mathrm{~ms}$ vs no urge $202 \pm 37 \mathrm{~ms}, t_{64}=2.07 ; p_{\text {Holm }}=0.021$, Cohen's $d: 0.44$ ), indicating that moderate urge improves inhibitory control. However, we did not find a difference in Stroop interference between the high urge and no urge conditions (planned contrast, lower tailed, high urge $154 \pm 45 \mathrm{~ms}$ vs no urge $202 \pm 37 \mathrm{~ms}, t_{64}=1.20, p_{\text {Holm }}=0.12$, Cohen's d: 0.30).

TAB LE 2 Error rate of Stroop tasks in each Stroop type in each urge condition

\begin{tabular}{llll} 
& \multicolumn{2}{l}{ Stroop type } & \\
\cline { 2 - 4 } Urge condition & Incongruent & Congruent & Word naming \\
\hline No urge & $4.29 \%$ & $0.86 \%$ & $2.57 \%$ \\
\hline Moderate urge & $2.28 \%$ & $1.14 \%$ & $2.28 \%$ \\
\hline High urge & $4.86 \%$ & $0.57 \%$ & $1.71 \%$ \\
\hline
\end{tabular}

Note. Error rate was higher in the incongruent trials compared to congruent or word-naming trials. Further, the error rate did not differ between urge conditions. (Friedman's chi-square test, Stroop type: $Q=17.6 P=0.0001$, Urge condition: $Q=1.69 P=0.43$ ).

\subsection{2 | Low BIS group}

The personalized distension pressures were not associated with participants' Stroop interference in low BIS group $(P>0.05)$. Consistent with our hypothesis, urge condition did not influence cognitive control among people with low BIS. Specifically, we did not find significant differences, neither between the moderate urge and no urge conditions (planned contrast, lower tail, moderate urge $158 \pm 32 \mathrm{~ms}$ vs no urge $168 \pm 24 \mathrm{~ms}, t_{68}=0.34 p_{\text {Holm }}=0.37$, Cohen's $d: 0.08$ ), nor between the high urge and no urge conditions (planned contrast, high urge $178 \pm 27 \mathrm{~ms}$ vs no urge $168 \pm 24 \mathrm{~ms}, t_{68}=0.72$ $p_{\text {Holm }}=0.76$, Cohen's $d: 0.17$ ).

Figure 3 shows the Stroop interference scores as a function of urge conditions in both the high and the low BIS group.

Together, these results are consistent with our hypothesis that moderate defecatory urge improves inhibitory control ability only in people with high BIS.

\section{4 | Stroop word naming}

Contrary to the Stroop color-naming trials, the Stroop word-naming trials consisted of color words (eg, the words, RED, BLUE, and GREEN) displayed in black font. Specifically, in these trials, participants reacted only to the words, instead of font color. As expected, there was no effect of urge on response latencies in this word-naming task, which did not require conflict resolution (planned contrasts, two-tailed, high BIS group: moderate vs no urge $t_{68}=0.49$ $p_{\text {Holm }}=0.63$ Cohen's $d$ : 0.12 , high vs no urge $t_{68}=0.81 P=0.42$ Cohen's $d$ : 0.20 ; low BIS group: moderate vs no urge $t_{68}=1.04$ $p_{\text {Holm }}=0.30$ Cohen's $d: 0.25$, high vs no urge $t_{68}=0.76 p_{\text {Holm }}=0.45$ Cohen's d: 0.18).
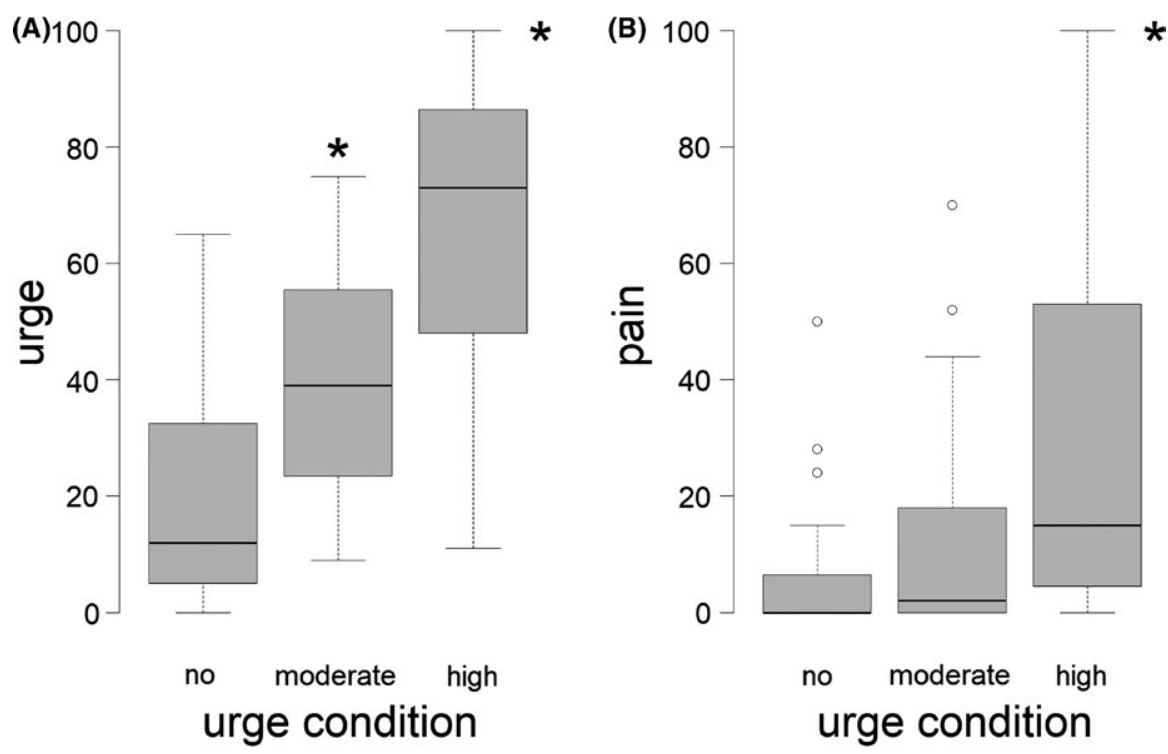

FIGURE 2 Rectal barostat successfully induced defecatory urge in both moderate and high urge conditions. Average VAS (A) urge scores in high and moderate urge conditions were significantly higher than in no urge condition $\left({ }^{*} P<0.05\right)$, and average VAS (B) pain scores in high urge condition was higher than in no urge condition $\left({ }^{*} P<0.05\right)$. VAS, visual analogue scale $(0-100 \mathrm{~mm})$ 


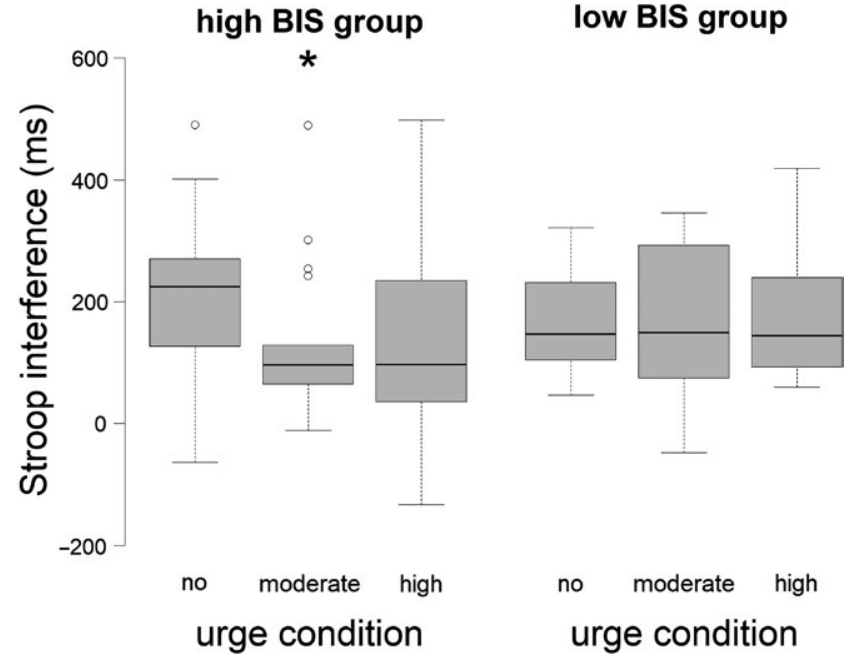

FIGURE 3 Stroop interference was smaller under moderate (but not high) urge condition, both compared to no urge condition, in the high BIS group $\left({ }^{*} P<0.05\right)$. The Stroop interference was not predicted by urge conditions in the low BIS group

\section{5 | Delay discounting task}

\subsection{1 | High BIS group}

As shown in Figure 4, participants preferred the larger-later reward more frequently under high urge compared to no urge $(z=2.06$, $P=0.039$, odds ratio $=1.51,95 \% \mathrm{Cl}: 1.02-2.25)$. However, participants' preference under moderate urge was not significantly different from their preference in the no urge condition $(z=0.11, P=0.91$, odds ratio $=1.02,95 \% \mathrm{Cl}: 0.73-1.42$ ). Overall, these results suggest that increased levels of urge render more patience in intertemporal choices, among people with high BIS.

\subsection{2 | Low BIS group}

As expected, urge conditions did not predict patience in intertemporal choices among participants with low BIS. Specifically, participants' preference under high urge was not significantly different from no urge $(z=-1.61, P=0.11$, odds ratio $=0.67,95 \% \mathrm{Cl}: 0.42-1.09)$, nor did their preference differ significantly between the moderate urge and no urge conditions $(z=-0.21, P=0.83$, odds ratio $=0.93,95 \%$ $\mathrm{Cl}$ : 0.50-1.75), as shown in Figure 4.

\section{4 | DISCUSSION}

Building on past findings suggesting the existence of a common, domain-independent inhibitory control system, we hypothesized and provided empirical evidence that inhibition of defecatory urge facilitates inhibitory control, across both cognitive and financial decisionmaking (ie, increased patience in the delay discounting) domains, among individuals with high BIS.

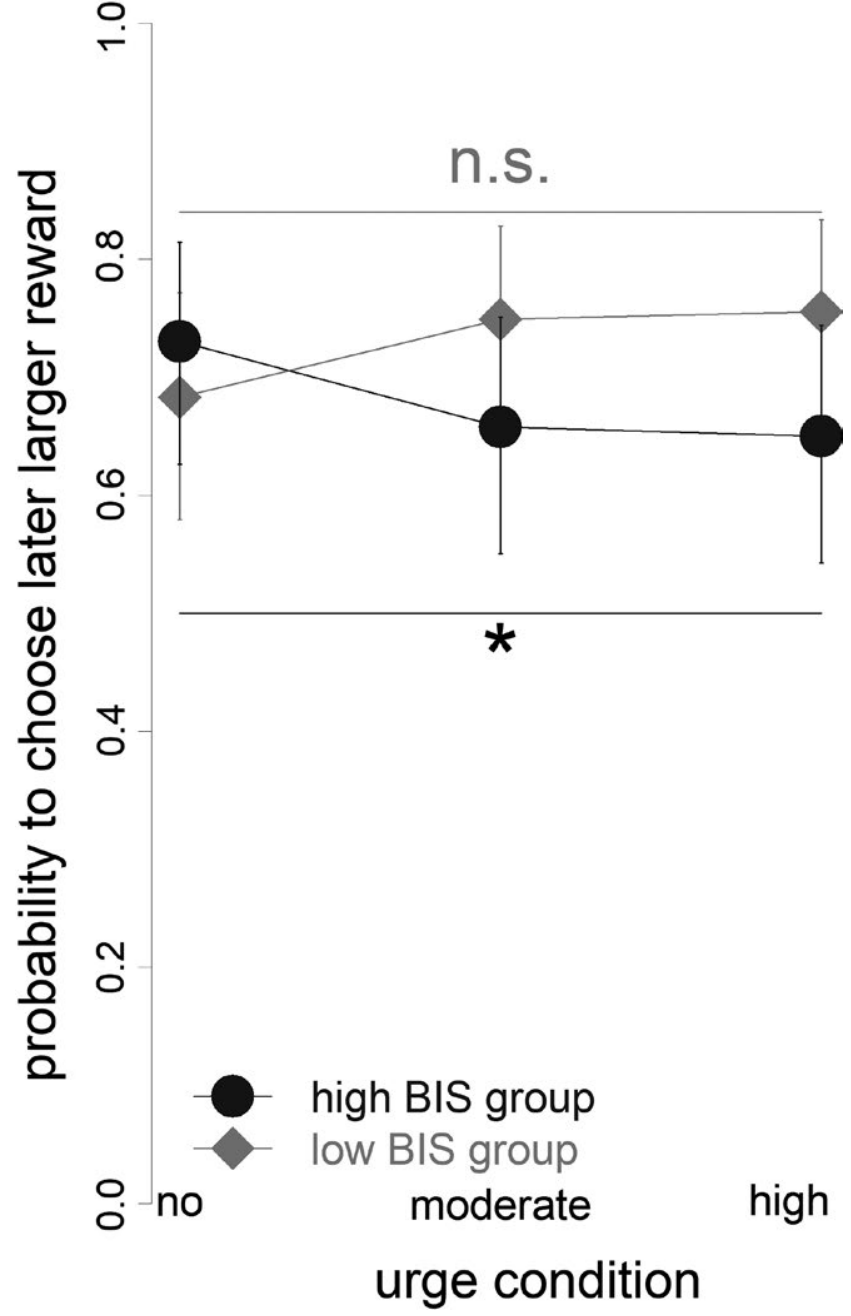

FIGURE 4 The probability that participants chose a larger-later reward, and thus show more patience, in the delay discounting task was higher under the high (but not moderate) urge condition, both compared to no urge condition, in the high BIS group $\left({ }^{*} P<0.05\right)$. Among participants with low BIS, urge condition did not significantly predict the choice of larger-later rewards

Particularly, our findings demonstrate that among participants with high BIS, moderate (but not high) urge significantly reduced Stroop interference, compared to a no urge condition. In other words, high BIS participants showed improved ability in inhibiting their automatic response tendencies under moderate defecatory urge induced by the rectal balloon. Moreover, we found that high BIS participants also showed more patience in the intertemporal monetary choices under high (but not moderate) urge, relative to no urge. Furthermore, in line with our reasoning, among individuals with low BIS, increased defecatory urge did not correspond to enhancement in the Stroop performance, nor did it increase patience in the delay discounting task. Overall, our findings are consistent with the inhibitory "spill-over" effect that was found earlier under an increased urge to urinate among the high BIS individuals. ${ }^{2}$

While our findings are consistent with the existence of a domaingeneral inhibitory control system, further research is necessary for 
explicating the neurological underpinnings of this system. From a neurological perspective, the anterior midcingulate cortex (aMCC) and adjacent pregenual anterior cingulate cortex (pACC) may be central to the inhibitory "spill-over" effects, given their important role in conflict detection. ${ }^{5,25}$ For example, pACC ${ }^{26,27}$ responds differentially to incongruent trials (ie, when the font color mismatches the word meaning) compared to congruent trials in the Stroop task, and those differential responses further extend to the posterior cingulate cortex. Moreover, the adjacent regions along the cingulate sulcus implement a domain-general inhibitory control process, ${ }^{28}$ and overlap partly with representations of other functions, such as pain, on the border between the aMCC and posterior midcingulate cortex. ${ }^{29}$ Importantly, a meta-analysis by Tillisch et al $^{30}$ revealed that the $\mathrm{pACC}$ and the aMCC were also activated in response to rectal balloon distension in healthy controls. It is, however, noteworthy that Tillisch et al focused on a very different research question, and designs of studies included in their meta-analysis were not identical to the present study. We, therefore, could only speculate which brain regions may be involved in the effects found in our study. It will be important to replicate the current findings using brain imaging techniques.

Furthermore, Amodio et al found a correlation between participants' BIS and pACC/aMCC responses to error detection in an electroencephalography study. ${ }^{31}$ More specifically, participants with higher BIS had stronger responses in the adjacent area between the pACC and aMCC when they performed a No-Go task, in which they were requested NOT to respond to a certain cue. ${ }^{31}$ In our study, the Stroop task, like the No-Go task, also required participants to inhibit their impulsive response tendencies. This might explain the differential effects in the high BIS and low BIS groups in our study. Putting the evidence together, we propose that the brain responses to rectal filling and inhibitory control overlap in the adjacent cingulate subregions including $\mathrm{pACC}$ and $\mathrm{AMCC}$, and that response in these areas are moderated by BIS. These regions may serve as an integrative center that mediated the "spill-over" effect observed in our study.

The delay discounting task consists of a series of choices between a smaller-sooner monetary reward and a larger-later reward. Therefore, the task is often used to measure the ability to control impulsiveness to maximize future outcomes. While Stroop performance and delay discounting both tap into self-regulation ability, and both are improved when people simultaneously control the urge to void, ${ }^{2}$ our findings suggest that the response to these tasks seems to be moderated by different levels of defecatory urge. Recent work has also found other dissociations between these tasks. ${ }^{32}$ Indeed, in the delay discounting task, brain regions that are related to self-regulation and inhibition were activated when participants made an impulsive choice, including the medial orbital frontal cortex, and medial prefrontal cortex (mPFC), ${ }^{1,14}$ but not including the abovementioned pACC or the aMCC, which are associated with inhibitory control. Further research needs to examine exactly why different tasks are differentially sensitive to urge level. One crucial difference might be that resisting to the lure of immediate financial rewards, and to wait for later large rewards, is a higher order "self-control" task which not only depends on people's ability to inhibit impulses but also depends on their subjective processing of delay times. ${ }^{33,34}$ Therefore, in contrast to the Stroop task, a delay discounting task might involve multiple cognitive operations, ${ }^{1}$ and is subject to individual differences in relative salience of saving and spending goals.

Another consideration is that the high urge condition was accompanied by increased pain experience, relative to the two other conditions. Pain could interrupt attention, ${ }^{35}$ and might do so more in a basic cognitive control task (eg, the Stroop task), compared to when people try to exert higher order, motivated, self-control (eg, in intertemporal choices). Notably, we used pain ratings as a covariate in all of our analysis, and it did not significantly influence any of the major outcomes. In addition to the effect induced by pain, extremely high urge might also undermine cognitive control. Although we did not extend our investigation to extreme, unbearable, high urges, prior research has found decrements in cognitive performance when the urge (to urinate) increased to a very high, unbearable, level. ${ }^{18}$ It is however noteworthy that the prior research had a small sample size $(n=8)$. Therefore, further investigation is necessary to confirm the differential cognitive responses to moderate and extremely high physiological urge conditions.

There are a few limitations to the current study. Firstly, the anticipation of visceral pain may influence inhibitory control. Therefore, we tried to minimize the effect of pain (and hence its anticipation) in our study design. Most of the volunteers reported no or minimal pain during distension, but some of them did. Hence, we controlled for pain ratings in our analyses. Further, we counterbalanced the order of distensions conditions in both tonic distension blocks and test blocks to minimize predictability. However, given the uncertainty about the order of distension intensities, we cannot completely rule out anticipatory effects, especially as we did not include ratings of anticipatory fear. Secondly, we limited our participants to the healthy normal weight population and did not include patients with functional bowel disorders such as irritable bowel syndrome (IBS), which is currently viewed as caused by dysregulation of the "braingut axis. ${ }^{36 "}$ Moreover, patients with IBS have shown increased sensitivity ${ }^{37,38}$ to rectal distension compared to normal controls, both in terms of urge and pain thresholds. Therefore, it would be interesting to investigate inhibitory control responses to different levels of rectal distension above and below the urge threshold of patients with IBS.

In summary, we found that inhibition of the urge to defecate facilitated inhibitory control and rendered more patience in the delay discounting task, among healthy humans with high BIS sensitivity. These findings have the potential to improve our understanding of the nature of different volitional cognitive control processes and their interaction.

\section{ACKNOWLEDGMENTS}

We would like to acknowledge the infrastructural support of Lieselot Holvoet in the Endoscopy Unit, University Hospital Gasthuisberg. We would also like to thank Jessica Biesiekierski and Julie Iven for 
language editing support, and Mark Phillip Keller for his technical assistance.

\section{DISCLOSURES}

The authors have nothing to disclose.

\section{AUTHOR CONTRIBUTIONS}

DZ, MC, NW, MM-J, MT, LW, and LVO designed the research; DZ and $\mathrm{MC}$ collected the data; DZ, MM-J, and LVO analyzed data and performed statistical analyses; DZ, MM-J, and LVO wrote the manuscript. MM-J, LW, JT, and MC revised the manuscript. LVO and LW had primary responsibility for the final content. All authors read and approved the final manuscript.

\section{ORCID}

Dongxing Zhao iD https://orcid.org/0000-0001-7477-7144

Maura Corsetti iD https://orcid.org/0000-0003-2957-4684

\section{REFERENCES}

1. Hofmann W, Schmeichel BJ, Baddeley AD. Executive functions and self-regulation. Trends Cogn Sci. 2012;16(3):174-180.

2. Tuk MA, Trampe D, Warlop L. Inhibitory spillover: increased urination urgency facilitates impulse control in unrelated domains. Psychol Sci. 2011;22(5):627-633.

3. Berkman ET, Burklund L, Lieberman MD. Inhibitory spillover: intentional motor inhibition produces incidental limbic inhibition via right inferior frontal cortex. Neurolmage. 2009;47(2):705-712.

4. Fenn E, Blandón-Gitlin I, Coons J, Pineda C, Echon R. The inhibitory spillover effect: Controlling the bladder makes better liars. Conscious Cogn. 2015;37:112-122.

5. Critchley HD. Neural mechanisms of autonomic, affective, and cognitive integration. J Comp Neurol. 2005;493(1):154-166.

6. MacDonald AW, Cohen JD, Stenger VA, Carter CS. Dissociating the role of the dorsolateral prefrontal and anterior cingulate cortex in cognitive control. Science. 2000;288(5472):1835-1838.

7. Todd S, Braver J. On the control of control: The role of dopamine in regulating prefrontal function and working. Paper presented at: MIT Press. Making Working Memory Work 2000.

8. Mertz H, Morgan V, Tanner G, et al. Regional cerebral activation in irritable bowel syndrome and control subjects with painful and nonpainful rectal distention. Gastroenterology. 2000;118(5):842-848.

9. Athwal BS, Berkley KJ, Hussain I, et al. Brain responses to changes in bladder volume and urge to void in healthy men. Brain. 2001;124(Pt 2):369-377.

10. Cohen JR, Lieberman MD. The common neural basis of exerting self-control in multiple domains. In: Hassin RR, Ochsner KN, Trope Y, eds. Self-control in society, mind, and brain, vol. 1. Oxford University Press; 2010:141-160.

11. Carver CS, White TL. Behavioral inhibition, behavioral activation, and affective responses to impending reward and punishment: the BIS/BAS scales. J Pers Soc Psychol. 1994;67(2):319.

12. Gray JA, McNaughton N. The neuropsychology of anxiety: An enquiry into the function of the septo-hippocampal system. Oxford University Press; 2003.
13. Corr PJ. Reinforcement sensitivity theory and personality. Neurosci Biobehav Rev. 2004;28(3):317-332.

14. McClure SM, Laibson DI, Loewenstein G, Cohen JD. Separate neural systems value immediate and delayed monetary rewards. Science. 2004;306(5695):503-507.

15. Stroop JR. Studies of interference in serial verbal reactions. J Exp Psychol. 1935;18(6):643.

16. Kane MJ, Engle RW. Working-memory capacity and the control of attention: the contributions of goal neglect, response competition, and task set to stroop interference. J Exp Psychol Gen. 2003;132(1):47-70.

17. Miyake A, Shah P. Models of working memory: Mechanisms of active maintenance and executive control. Cambridge, UK: Cambridge University Press; 1999.

18. Lewis MS, Snyder PJ, Pietrzak RH, Darby D, Feldman RA, Maruff P. The effect of acute increase in urge to void on cognitive function in healthy adults. Neurourol Urodyn. 2011;30(1):183-187.

19. Basilisco G, De Marco E, Tomba C, Cesana BM. Bowel urgency in patients with irritable bowel syndrome. Gastroenterology. 2007;132(1):38-44.

20. Kwan CL, Mikula K, Diamant NE, Davis KD. The relationship between rectal pain, unpleasantness, and urge to defecate in normal subjects. Pain. 2002;97(1-2):53-63.

21. Floden D, Vallesi A, Stuss DT. Task context and frontal lobe activation in the Stroop task. J Cogn Neurosci. 2011;23(4):867-879.

22. Kirby KN, Petry NM, Bickel WK. Heroin addicts have higher discount rates for delayed rewards than non-drug-using controls. J Exp Psychol Gen. 1999;128(1):78.

23. Selst MV, Jolicoeur P. A solution to the effect of sample size on outlier elimination. Exp Psychol. 1994;47(3):631-650.

24. Jones J, Sullivan PS. Impulsivity as a risk factor for HIV transmission in men who have sex with men: a delay discounting approach. $J$ Homosex. 2015;62(5):588-603.

25. Lieberman MD, Eisenberger NI. The dorsal anterior cingulate cortex is selective for pain: results from large-scale reverse inference. Proc Natl Acad Sci U S A. 2015;112(49):15250-15255.

26. Badzakova-Trajkov G, Barnett KJ, Waldie KE, Kirk IJ. An ERP investigation of the stroop task: the role of the cingulate in attentional allocation and conflict resolution. Brain Res. 2009;1253:139-148.

27. Carter CS, van Veen V. Anterior cingulate cortex and conflict detection: an update of theory and data. Cogn Affect Behav Neurosci. 2007;7(4):367-379.

28. Shackman AJ, Salomons TV, Slagter HA, Fox AS, Winter JJ, Davidson RJ. The integration of negative affect, pain, and cognitive control in the cingulate cortex. Nat Rev Neurosci. 2011;12(3):154-167.

29. Kragel PA, Kano M, Van Oudenhove L, et al. Generalizable representations of pain, cognitive control, and negative emotion in medial frontal cortex. Nat Neurosci. 2018;21(2):283.

30. Tillisch K, Mayer EA, Labus JS. Quantitative meta-analysis identifies brain regions activated during rectal distension in irritable bowel syndrome. Gastroenterology. 2011;140(1):91-100.

31. Amodio DM, Master SL, Yee CM, Taylor SE. Neurocognitive components of the behavioral inhibition and activation systems: implications for theories of self-regulation. Psychophysiology. 2008;45(1):11-19.

32. Scherbaum S, Frisch S, Holfert AM, O'Hora D, Dshemuchadse M. No evidence for common processes of cognitive control and selfcontrol. Acta Psychol. 2017;182:194-199.

33. Zauberman G, Kim BK, Malkoc SA, Bettman JR. Discounting time and time discounting: subjective time perception and intertemporal preferences. J Mark Res. 2009;46(4):543-556.

34. Kim BK, Zauberman G. Perception of anticipatory time in temporal discounting. J Neurosci Psychol Econ. 2009;2(2):91. 
35. Eccleston C, Crombez G. Pain demands attention: a cognitive-affective model of the interruptive function of pain. Psychol Bull. 1999;125(3):356-366.

36. Kennedy PJ, Clarke G, O'Neill A, et al. Cognitive performance in irritable bowel syndrome: evidence of a stress-related impairment in visuospatial memory. Psychol Med. 2014;44:1553-1566.

37. Tornblom H, Van Oudenhove L, Tack J, Simren M. Interaction between preprandial and postprandial rectal sensory and motor abnormalities in IBS. Gut. 2014;63(9):1441-1449.

38. Zar S, Benson MJ, Kumar D. Rectal afferent hypersensitivity and compliance in irritable bowel syndrome: differences between diarrhoea-predominant and constipation-predominant subgroups. Eur J Gastroenterol Hepatol. 2006;18(2):151-158.

\section{SUPPORTING INFORMATION}

Additional supporting information may be found online in the Supporting Information section at the end of the article.

How to cite this article: Zhao D, Corsetti M, Moeini-Jazani M, et al. Defecatory urge increases cognitive control and intertemporal patience in healthy volunteers.

Neurogastroenterol Motil. 2019;e13600. https://doi. org/10.1111/nmo.13600 